\title{
Effect of coronal structure on loop oscillations: exponential profiles
}

\author{
A. J. Díaz ${ }^{\star}$, G. R. Donnelly, and B. Roberts \\ Mathematical Institute, University of St Andrews, St Andrews, KY16 9SS, Scotland, UK \\ e-mail: [antonio;gavin; bernie]@mcs.st-and.ac.uk \\ Received 31 July 2007 / Accepted 18 September 2007

\begin{abstract}
Aims. The role of longitudinal structuring of the surrounding corona on the modes of oscillation of a coronal magnetic flux tube was studied in Donnelly et al. (2006) for a piecewise uniform profile. Here we investigate whether a more realistic continuous exponential profile changes the conclusions drawn from that paper.

Methods. A partial differential equation is derived for the total pressure perturbation of the fast modes, which is then decomposed by separation of variables. The longitudinal part is solved numerically, obtaining a dispersion relation. These results are supported by an analytical investigation in terms of Bessel functions of purely imaginary order.

Results. Structure in the interior of the loop shifts the frequencies of the modes (and may trap higher harmonics), an effect which can be understood by taking an averaged profile with a suitable weight. Structure in the environment modifies only slightly the frequencies, but displaces the cutoff frequency. The shift due to the structure in the fundamental period is small, but the ratio between the periods of the fundamental mode and its harmonics can be used to probe the structure.

Conclusions. The results support our previous study in a more realistic, continuously varying profile and provide limits to the conclusions drawn in coronal seismology if an unstructured loop is used. Also, the ratio between the period of the fundamental kink (even) mode and its first (odd) harmonic is proven as an extra seismological tool for coronal loops.
\end{abstract}

Key words. Sun: oscillations - Sun: magnetic fields - Sun: corona

\section{Introduction}

It is clear from the abundance of observations that fast magnetohydrodynamic (MHD) oscillations are a commonly occurring event for a coronal loop. As with any wave mode, oscillations of coronal loops carry information about the medium in which the wave propagates. If we understand the wave mode and its effect on the plasma as it propagates, we can extract the information the wave possesses about the plasma; this is known as coronal seismology (Roberts et al. 1984; Roberts 1986; Nakariakov et al. 1999; Nakariakov \& Ofman 2001; De Moortel et al. 2002; Andries et al. 2005a,b; McEwan et al. 2006).

In order to carry out coronal seismology, a good understanding of the oscillatory modes of a structure is necessary. Models generally consist of a straight cylindrical flux tube which is either infinitely long or line-tied (finite length) (Edwin \& Roberts 1983; Roberts et al. 1984). When line-tying is considered it is also possible to study the effect of longitudinal structuring (Nakariakov et al. 2000; Nakariakov \& Ofman 2001; James 2003; Mendoza-Briceño et al. 2004; Díaz et al. 2002, 2004, 2006; Andries et al. 2005a,b; Donnelly et al. 2006; McEwan et al. 2006; Dymova \& Ruderman 2006; Erdélyi \& Verth 2007). In particular, in Donnelly et al. (2006) step functions are used to represent dense chromospheric layers both inside and outside the loop. However, the use of step functions is only a first approximation and, in an actual coronal loop, a continuously varying density profile is expected.

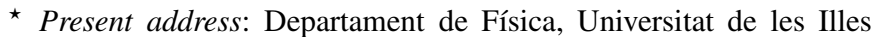
Balears, 07122 Palma de Mallorca, Spain.
In this paper we consider continuous longitudinal density profiles, comparing our results with the uniform line-tied loop. We consider a loop of finite radius. An alternative approach is to use the thin tube approximation (Dymova \& Ruderman 2006; Erdélyi \& Verth 2007; McEwan et al. 2007). First we consider a loop with exponential behaviour along its full length, for which the influence of the loop radius as well as the level of structure between the loop apex and footpoint is studied. Secondly, a loop with uniform coronal region but with exponentially varying chromospheric regions close to the footpoints is considered.

We use the same mathematical method as in Donnelly et al. (2006), adapted to permit different longitudinal dependences in the density and Alfvén profiles. Since these profiles are continuous, a numerical approach may also be used to solve the resulting wave equations.

\section{Equilibrium model and wave equations}

We consider a spatially structured static equilibrium of a cylindrically symmetric line-tied coronal magnetic flux tube (lying parallel to the $z$ axis) of length $2 L$ and radius $a$. The equilibrium magnetic field $\boldsymbol{B}_{0}=B_{0} \boldsymbol{e}_{z}$ is assumed to be uniform throughout the medium and is aligned with the loop. The equilibrium density $\rho_{0}$ is structured both radially and longitudinally. Gravitational effects are ignored.

Small amplitude oscillations about this equilibrium are considered in the limit of a zero $\beta$ plasma, in which wave propagation is dominated by magnetism and slow waves are absent. The ideal adiabatic MHD equations in cylindrical coordinates $(r, \theta, z)$ 
reduce to the following system of coupled partial differential equations for a zero $\beta$ plasma (Díaz et al. 2002; Díaz 2004; Díaz et al. 2006)

$\rho_{0}\left[\frac{\partial^{2}}{\partial t^{2}}-c_{\mathrm{A}}^{2}(z) \frac{\partial^{2}}{\partial z^{2}}\right] v_{r}=-\frac{\partial^{2} p_{\mathrm{T}}}{\partial r \partial t}$,

$\left[\frac{\partial^{2}}{\partial t^{2}}-c_{\mathrm{A}}^{2}(z) \nabla^{2}\right] p_{\mathrm{T}}=0$,

where $c_{\mathrm{A}}(z)=B_{0} / \sqrt{\mu \rho_{0}}$ is the Alfvén and speed, $v_{r}$ the radial component of the perturbed flow and $p_{\mathrm{T}}$ the perturbed total pressure. Equations (1)-(2) apply in each region (coronal and chromospheric) of the flux tube and also in its environment.

We allow for a general longitudinal and transverse structuring in the form of step functions, the equilibrium plasma density profile taking the form

$\rho_{0}(r, z)= \begin{cases}\rho_{\mathrm{i}}(z), & r<a, \\ \rho_{\mathrm{e}}(z), & r>a,\end{cases}$

where we denote internal and external equilibrium values by the subscripts " $i$ " and "e" respectively.

We consider trapped modes for which there is no propagation of energy towards or away from the loop. This is achieved by imposing $v_{r} \rightarrow 0$ and $p_{\mathrm{T}} \rightarrow 0$ as $r \rightarrow \infty$. The loop is linetied with the footpoints fixed in the dense photosphere, where it is expected that coronal perturbations carry such small energy that they are incapable of perturbing the dense lower layers (Hood 1986): this leads to the line-tying boundary condition $v_{r}(z= \pm L)=0$. In this model, we have interfaces over which the equilibrium plasma properties jump discontinuously so we must know how the perturbations behave across such surfaces. In the case where the interface is parallel to the equilibrium field, the boundary conditions are (Goedbloed 1983; Díaz et al. 2001; Díaz 2004)

$\hat{\boldsymbol{n}} \cdot[\boldsymbol{v}]=\hat{\boldsymbol{n}} \cdot[\boldsymbol{B}]=0, \quad\left[p_{\mathrm{T}}\right]=0$,

were $\hat{\boldsymbol{n}}$ is the vector normal to the surface and $\boldsymbol{v}$ denotes the perturbed velocity. Thus, the perturbed pressure $p_{\mathrm{T}}$ and the components of the perturbed velocity and magnetic field perpendicular to the interface are required to be continuous. For interfaces which are not parallel to the equilibrium magnetic field, the boundary conditions are

$[\boldsymbol{v}]=[\boldsymbol{B}]=\left[p_{\mathrm{T}}\right]=0 ;$

in this case all components of the velocity and the magnetic field, as well as the total pressure, must be continuous at the interface.

\section{Analytical solution}

We study oscillatory solutions to Eqs. (1) and (2). Following Díaz et al. (2002), we assume a solution which is separable in space and time, writing

$p_{\mathrm{T}}(r, \theta, z, t)=u(r) \Phi(\theta) h(z) \exp (i \omega t)$,

with $\omega$ the frequency. This form for the total pressure perturbations $p_{\mathrm{T}}$ produces a set of ordinary differential equations:

$\frac{\mathrm{d}^{2} u}{\mathrm{~d} r^{2}}+\frac{1}{r} \frac{\mathrm{d} u}{\mathrm{~d} r}-\left(\lambda^{2}+\frac{m^{2}}{r^{2}}\right) u=0$,

$\frac{\mathrm{d}^{2} \Phi}{\mathrm{d} \theta^{2}}+m^{2} \Phi=0$ and

$$
\frac{\mathrm{d}^{2} h}{\mathrm{~d} z^{2}}+\left(\frac{\omega^{2}}{c_{\mathrm{Aj}}^{2}(z)}+\lambda^{2}\right) h=0, \quad j=i \quad \text { or } \quad e,
$$

where $m^{2}$ and $\lambda^{2}$ are the separation constants. Again, internal $(r<a)$ quantities are denoted by a subscript "i" and external $(r>a)$ quantities by a subscript "e".

Solving Eq. (8) for $\Phi$ gives

$\Phi(\theta)=\Phi(0) \exp (\operatorname{im} \theta)$.

This solution applies in both the tube interior and the exterior, since the total pressure must be continuous across the tube boundary. The periodicity in the $\theta$-direction implies that $m$ must be an integer, the same for both the interior and exterior regions.

Equation (7) has solutions in the form

$u(r)=\left\{\begin{array}{l}A_{1} I_{m}\left(\lambda^{\mathrm{i}} r\right), \quad r \leq a, \\ A_{2} K_{m}\left(\lambda^{\mathrm{e}} r\right), \quad r>a,\end{array}\right.$

where $I_{m}$ and $K_{m}$ denote modified Bessel functions (Abramowitz \& Stegun 1964) and $A_{1}$ and $A_{2}$ are arbitrary constants. We have required the solution to be finite in the region $r<a$ and that all perturbations decline to zero as $r \rightarrow \infty$, requiring $\lambda^{\mathrm{e}}>0$. The frequency, $\omega_{\text {cut }}$, at which $\left(\lambda^{\mathrm{e}}\right)^{2}$ changes sign defines the cutoff frequency, marking the transition between trapped and leaky modes. For a homogeneous loop embedded in a homogeneous environment, the resultant cutoff frequency for the first family of even modes is (Roberts et al. 1984)

$\omega_{\mathrm{cut}}=\pi c_{\mathrm{Ae}} / 2 L$

Finally, we consider the equation for $h(z)$, which defines the longitudinal structuring. We can proceed generally, without specifying the equilibrium density profile, by noting that for certain choices of Alfvén profile the differential operator

$$
\frac{\mathrm{d}^{2}}{\mathrm{~d} z^{2}}+\frac{\omega^{2}}{c_{\mathrm{A}}^{2}(z)}+\lambda^{2}
$$

is self-adjoint. If this is the case and $h(z= \pm L)=0$, which is equivalent to the line-tying condition $v_{r}(z= \pm L)=0$, then the Sturm-Liouville theorem can be applied with the implication that the set of solutions to Eq. (9) form a complete set on $-L \leq z \leq L$. This allows the external solutions $h_{n}^{\mathrm{e}}(z)$ to be expanded in terms of the complete set of internal solutions $h_{m}^{\mathrm{i}}(z)$ (Díaz et al. 2001):

$$
h_{n}^{\mathrm{e}}(z)=\sum_{m=1}^{\infty} H_{n m} h_{m}^{\mathrm{i}}(z)
$$

where the coefficients $H_{n m}$ are given by

$H_{n m}=\int_{-L}^{L} h_{n}^{\mathrm{e}}(z) h_{m}^{\mathrm{i}}(z) \mathrm{d} z$

In order to satisfy the continuity of $p_{\mathrm{T}}$ and $v_{r}$ on the tube interface $r=a$, we write $p_{\mathrm{T}}$ as a linear combination of all solutions (Díaz et al. 2001):

$p_{\mathrm{T}}(r, \theta, z)=\Phi(\theta) \begin{cases}\sum_{n=1}^{\infty} u_{n}^{\mathrm{i}}(r) h_{n}^{\mathrm{i}}(z), & r<a, \\ \sum_{n=1}^{\infty} u_{n}^{\mathrm{e}}(r) h_{n}^{\mathrm{e}}(z), & r>a .\end{cases}$ 
Hence, we find from Eq. (1)

$v_{r}(r, \theta, z)=\Phi(\theta) \frac{-i \omega}{\rho_{\mathrm{i}} c_{\mathrm{Ai}}^{2}}\left\{\begin{array}{l}\sum_{n=1}^{\infty} \frac{1}{\left(\lambda_{n}^{\mathrm{i}}\right)^{2}} \frac{\mathrm{d} u_{n}^{\mathrm{i}}}{\mathrm{d} r} h_{n}^{\mathrm{i}}(z), r<a, \\ \sum_{n=1}^{\infty} \frac{1}{\left(\lambda_{n}^{\mathrm{e}}\right)^{2}} \frac{\mathrm{d} u_{n}^{\mathrm{e}}}{\mathrm{d} r} h_{n}^{\mathrm{e}}(z), r>a .\end{array}\right.$

From the continuity of $p_{\mathrm{T}}$ and $v_{r}$ we can derive the set of linear equations for the amplitude coefficients $C_{s}$ that arise in the solution of Eq. (11): allowing that $\left(\lambda_{n}^{\mathrm{i}}\right)^{2}$ may change sign after a finite number $s=l$ terms, these relations are

$$
\begin{gathered}
\sum_{s=1}^{l} H_{s n}\left[\frac{1}{\lambda_{n}^{\mathrm{i}}} I_{m}^{\prime}\left(\lambda_{n}^{\mathrm{i}} a\right) K_{m}\left(\lambda_{s}^{\mathrm{e}} a\right)-\frac{1}{\lambda_{s}^{\mathrm{e}}} I_{m}\left(\lambda_{n}^{\mathrm{i}} a\right) K_{m}^{\prime}\left(\lambda_{s}^{\mathrm{e}} a\right)\right] C_{s} \\
+\sum_{s=l+1}^{\infty} H_{s n}\left[\frac{1}{\left\{\lambda_{n}^{\mathrm{i}}\right\}^{\star}} J_{m}^{\prime}\left(\left\{\lambda_{n}^{\mathrm{i}}\right\}^{\star} a\right) K_{m}\left(\lambda_{s}^{\mathrm{e}} a\right)+\right. \\
\left.\frac{1}{\lambda_{s}^{\mathrm{e}}} J_{m}\left(\left\{\lambda_{n}^{\mathrm{i}}\right\}^{\star} a\right) K_{m}^{\prime}\left(\lambda_{s}^{\mathrm{e}} a\right)\right] C_{s}=0 .
\end{gathered}
$$

Here the star denotes the imaginary part of the purely imaginary number $\lambda_{n}^{\mathrm{i}}: \lambda_{n}^{\mathrm{i}}=i\left\{\lambda_{n}^{\mathrm{i}}\right\}^{\star}$.

The dispersion relation for the trapped modes of oscillation of a line-tied coronal loop with arbitrary longitudinal environmental structuring and step functions in the radial coordinate is satisfied when the determinant of the system of Eq. (18) vanishes. This condition describes the sausage $(m=0), \operatorname{kink}(m=1)$ and fluting $(m \geq 2)$ modes. An interesting aspect of this general dispersion relation is that, due to the summation over all harmonics, each mode is in general a combination of both surface and body modes, whereas in a homogeneous tube these may be considered separately.

\subsection{Uniform loop and environment}

The modes of oscillation of a uniform loop embedded in a uniform environment are governed by the dispersion relation (see Edwin \& Roberts 1983)

$\frac{1}{\lambda_{n}^{\mathrm{e}}} \frac{K_{m}^{\prime}\left(\lambda_{n}^{\mathrm{e}} a\right)}{K_{m}\left(\lambda_{n}^{\mathrm{e}} a\right)}=\frac{1}{\lambda_{n}^{\mathrm{i}}} \frac{J_{m}^{\prime}\left(\lambda_{n}^{\mathrm{i}} a\right)}{J_{m}\left(\lambda_{n}^{\mathrm{i}} a\right)}$

where

$$
\left[\lambda_{n}^{\mathrm{i}}\right]^{2}=M_{n}^{2}-\frac{\omega^{2}}{c_{\mathrm{Ai}}^{2}}, \quad\left[\lambda_{n}^{\mathrm{e}}\right]^{2}=M_{n}^{2}-\frac{\omega^{2}}{c_{\mathrm{Ae}}^{2}}
$$

for even modes,

$M_{n}^{2}=\frac{(2 n-1) \pi}{2 L} \quad n=1,2,3 \ldots$

Modes of different longitudinal wavenumbers are denoted by a subscript $n$. This dispersion relation follows from Eq. (18) by noticing that if a uniform loop and environment is considered then $H_{s n}=\delta_{s n}$, the Kronecker delta. So in the absence of longitudinal structure families with different longitudinal wavenumbers are decoupled and the cutoff frequency is different for each family, occurring when

$\omega=\omega_{\mathrm{cut}}=\frac{(2 n-1) \pi c_{\mathrm{Ae}}}{2 L}$.

We will only be concerned with modes lying below the first $(n=1)$ cutoff frequency as the introduction of longitudinal structuring results in the tube supporting a superposition of these families and the resulting set of modes has a cutoff frequency corresponding to $\left(\lambda_{1}^{\mathrm{e}}\right)^{2}=0$. Note that in some cases the uniform
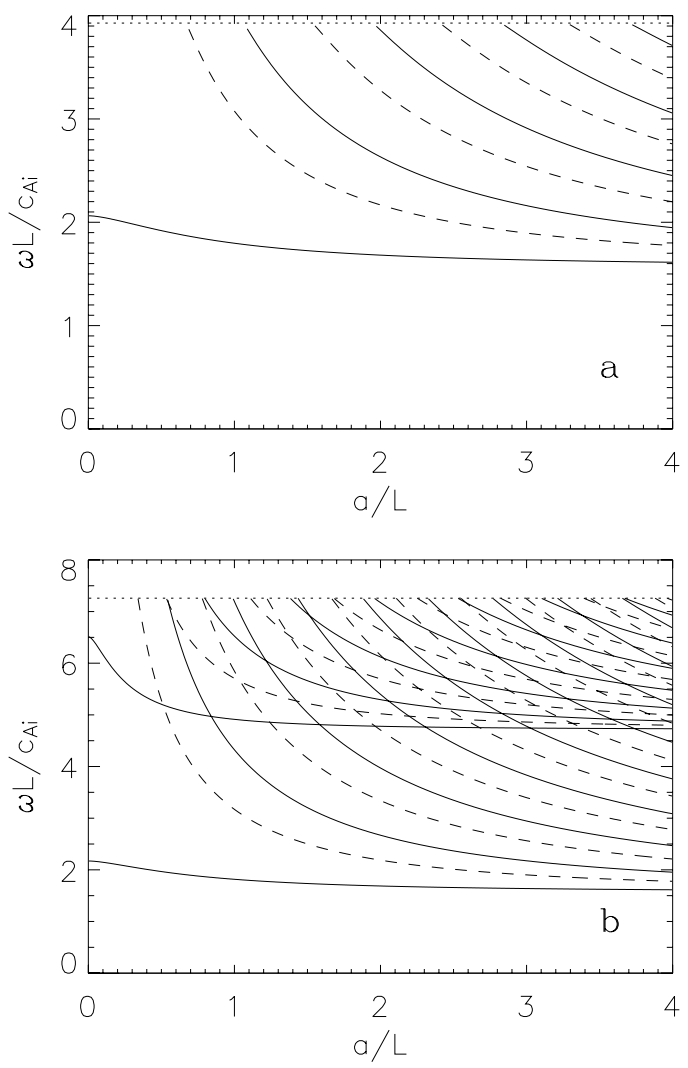

Fig. 1. Dispersion diagram plotting non-dimensional frequency $\omega L / c_{\mathrm{Ai}}$ against the non-dimensional loop radius $a / L$ for a uniform loop of length $2 L$ and a uniform environment, with a) $c_{\mathrm{Ae}}=2.5 c_{\mathrm{Ai}}$ and b) $c_{\mathrm{Ae}}=$ $4.62 c_{\mathrm{Ai}}$. Sausage modes are shown as dashed curves and kink modes as solid curves; the cutoffs are shown as dotted horizontal lines.

loop model will support a second family (having 3 extrema in the $z$ direction) with frequencies less than the cutoff frequency of the first family in the range of $a / L=0-4$. This will become important in the structured case as it appears to give an indication of when mode coupling can occur.

Figure 1 shows the dispersion diagram plotting nondimensional frequency $\omega L / c_{\mathrm{Ai}}$ against $a / L$ for a uniform loop and uniform environment, with (a) $c_{\mathrm{Ae}}=2.5 c_{\mathrm{Ai}}$ or (b) $c_{\mathrm{Ae}}=$ $4.62 c_{\mathrm{Ai}}$. This diagram is similar to the fast mode band of the coronal case considered Edwin \& Roberts (1983), with the fundamental kink mode the only mode to propagate for all $a / L$. The kink mode has a phase speed $\omega / M_{1} \approx c_{\mathrm{k}}$ in the thin tube limit, with

$c_{\mathrm{k}}=c_{\mathrm{Ai}} \sqrt{2 /\left(1+c_{\mathrm{Ai}^{2}} / c_{\mathrm{Ae}^{2}}\right)}$;

thus

$\frac{\omega L}{c_{\mathrm{Ai}}}=\frac{\pi}{2} \sqrt{\frac{2}{1+c_{\mathrm{Ai}^{2}} / c_{\mathrm{Ae}^{2}}}}$.

All other modes reach the cutoff frequency $\omega_{\text {cut }} L / c_{\mathrm{Ai}}=$ $\pi c_{\mathrm{Ae}} / 2 c_{\mathrm{Ai}}$. We have plotted modes that give an even disturbance about the $z=0$ plane; the odd modes cause odd disturbances about the $z=0$ plane and have a cutoff frequency which is twice that of the even modes (in the situation where the environment is uniform), $\omega_{\text {cut }} L / c_{\mathrm{Ai}}=\pi c_{\mathrm{Ae}} / c_{\mathrm{Ai}}$. Notice that the cutoff frequency is determined entirely by the loop exterior. Fluting modes $(m \geq 2)$ are also described by the dispersion relation but have not been plotted in Fig. 1. All the modes are seen to be 
highly dispersive, apart from the fundamental kink mode in the realistic thin tube region of the diagram, $a / L=10^{-3}-10^{-1}$ where its frequency is insensitive to the loop ratio $a / L$.

Figure 1b shows an example of the dispersion diagram with higher density contrast between the interior and exterior, where both the first and second $n$ families appear below the $n=1$ cutoff frequency. The first family, which also appears in Fig. 1a, has one extremum in the longitudinal direction while the second even family has three extrema in the longitudinal direction, so they represent different longitudinal harmonics. As there is no longitudinal structuring of the loop or the environment each family, with different longitudinal wavenumbers are decoupled; this permits the crossing of their dispersion curves.

\section{Exponential profiles}

We introduce longitudinal structure in the loop considering an exponential Alfvén profile of the form

$c_{\mathrm{Ai}}(z)=c_{\mathrm{Ai}}(0) \exp \left(-\alpha_{\mathrm{i}}|z| / L\right)$,

$c_{\mathrm{Ae}}(z)=c_{\mathrm{Ae}}(0) \exp \left(-\alpha_{\mathrm{e}}|z| / L\right)$

where $\alpha_{\mathrm{j}}$, with $j=i$ or e, governs the fall-off rate between the apex $(z=0)$ and the loop footpoints $(z= \pm L)$. Notice that all the profiles discussed in this paper are symmetric around the $z=$ 0 plane, so we classify the solutions in even and odd modes, and we concentrate in the region with $0 \leq z \leq L$. Using symmetric profiles means also that at the apex point $z=0$ there is a cusp. With the exponential Alfvén profile in Eq. (25), Eq. (9) becomes

$\frac{\mathrm{d}^{2} h}{\mathrm{~d} z^{2}}+\left(\frac{\omega^{2}}{c_{\mathrm{Aj}}^{2}} \exp \left(2 \alpha_{\mathrm{j}} z / L\right)+\lambda_{n}^{2}\right) h=0$.

We may transform Eq. (26) into a Bessel equation for either purely real or purely imaginary order (depending upon whether $\lambda_{n}^{2} \geq 0$ or $\lambda_{n}^{2}<0$, respectively) using the change of variable

$x_{\mathrm{j}}=\frac{L \omega}{\alpha_{\mathrm{j}} c_{\mathrm{Aj}}} \exp \left(\alpha_{\mathrm{j}} z / L\right)$

the result is

$\frac{\mathrm{d}^{2} h}{\mathrm{~d} x_{\mathrm{j}}^{2}}+\frac{1}{x_{\mathrm{j}}} \frac{\mathrm{d} h}{\mathrm{~d} x_{\mathrm{j}}}+\left(1+\frac{\lambda_{n}^{2} L^{2}}{\alpha_{\mathrm{j}}^{2} x_{\mathrm{j}}^{2}}\right) h=0$.

Equation (28) has solutions

$h(z)= \begin{cases}B_{1} J_{v}\left(x_{\mathrm{j}}\right)+B_{2} Y_{v}\left(x_{\mathrm{j}}\right), & \lambda_{n}^{2} \geq 0, \\ B_{3} J_{i v}\left(x_{\mathrm{j}}\right)+B_{4} Y_{i v}\left(x_{\mathrm{j}}\right), & \lambda_{n}^{2}<0,\end{cases}$

where $v=\left|\lambda_{n} L / \alpha_{\mathrm{j}}\right| ; B_{1}, B_{2}, B_{3}$ and $B_{4}$ are arbitrary constants. Notice that these solutions are a linear combination of ordinary Bessel functions; however, their order is generally non-integer and indeed may be purely imaginary (which implies that the Bessel functions are not necessarily real-valued). We are interested in real valued solutions to Eq. (28). Therefore, in the case $\lambda_{n}^{2}<0$ we define two linearly independent real-valued solutions to Eq. (28) as (Dunster 1990)

$F_{i v}\left(x_{\mathrm{j}}\right)=\frac{1}{2} \sec \left(\frac{i v \pi}{2}\right)\left\{J_{i v}\left(x_{\mathrm{j}}\right)+J_{-i v}\left(x_{\mathrm{j}}\right)\right\}$

and

$G_{i v}\left(x_{\mathrm{j}}\right)=\frac{1}{2} \operatorname{cosec}\left(\frac{i v \pi}{2}\right)\left\{J_{i v}\left(x_{\mathrm{j}}\right)-J_{-i v}\left(x_{\mathrm{j}}\right)\right\}$.
The functions $F_{i v}$ and $G_{i v}$ can be used to write down appropriate real solutions to Eq. (28) in the case of $\lambda_{n}^{2}<0$.

Although the ordinary Bessel function has been extensively explored in the case of real orders the properties and methods of calculation of Bessel functions of purely imaginary order are not well known and the use of recurrence relations or integral representations is not straightforward (Dunster 1990). Accordingly, we find the use of numerical methods to solve the equation directly a more convenient approach for determining solutions of Eq. (26), although the solution in Eq. (29) provides a useful guide for understanding the properties of the solutions.

\subsection{Structured loop}

We may isolate the effect of loop structure. Previous studies in this area have considered loop profiles using step functions (Díaz et al. 2004) to approximate a coronal region above a dense chromospheric layer with a homogeneous environment or profiles coming from other hydrodynamic equilibria (Andries et al. 2005b; Díaz et al. 2006). Recently, continuous profiles have also been studied by solving analytically and numerically the $1 \mathrm{D}$ equation that it is obtained in the thin-tube limit (Dymova \& Ruderman 2006; Erdélyi \& Verth 2007; McEwan et al. 2007). We extend these models by approximating the longitudinal density structure by a continuous exponential profile. A density increase due to structure results in a decrease of the oscillation frequency (which may lead to trapping of more modes) but since the cutoff frequency depends purely on the structure of the external medium, it remains at $\omega_{\text {cut }} L / c_{\mathrm{Ai}}=\pi c_{\mathrm{Ae}} / 2 c_{\mathrm{Ai}}$.

Figure $2 \mathrm{a}$ shows the dispersion diagram for the case $\rho_{\mathrm{i}}(L) / \rho_{\mathrm{i}}(0)=1.6$. The diagram is similar in structure to that of Edwin \& Roberts (1983) with only the fundamental kink mode propagating for all values of $a / L$ and all other modes reaching the cutoff frequency where these modes become leaky. All modes are highly dispersive and sausage and kink modes are distributed alternately; in the thin tube limit the frequency of the fundamental kink mode is insensitive to $a / L$. Comparing Fig. 2 a with Fig. 1a, we see the internal structure has resulted in a decrease in the frequency of each mode. This is illustrated by the modification of the kink mode from $\omega L / c_{\mathrm{Ai}}=2.1$ (Eq. (24)) in the uniform case to $\omega L / c_{\mathrm{Ai}}=1.9$ as a result of the exponential structure.

We also consider, in Fig. 2b, a density structure of $\rho_{\mathrm{i}}(L) / \rho_{\mathrm{i}}(0)=8$. The structure of the dispersion diagram has now significantly altered, with many more modes propagating in a given range of $a / L$. Not only the fundamental kink mode but also its first harmonic now propagate as trapped modes for all $a / L$ (compare with Fig. 1b); the frequency of the fundamental mode has retained its insensitivity to $a / L$ in the thin tube limit but the first harmonic does not share this property; all other modes reach the cutoff frequency. The structure of one sausage mode lying between consecutive kink modes has now been broken as a result of many mode interactions at avoided crossings (the spatial structure across an avoided crossing will be discussed later) between like curves. Also, the presence of avoided crossings seems to allow sausage and kink modes to cross, and so have the same frequency for a specific loop dimension.

\subsubsection{Approximation by a weighted uniform profile}

The modifications in the frequency in Fig. 2 are mainly due to an uniform scaling of the dispersion curves as seen in Fig. 3, where the dispersion curves of a uniform loop embedded in a uniform 

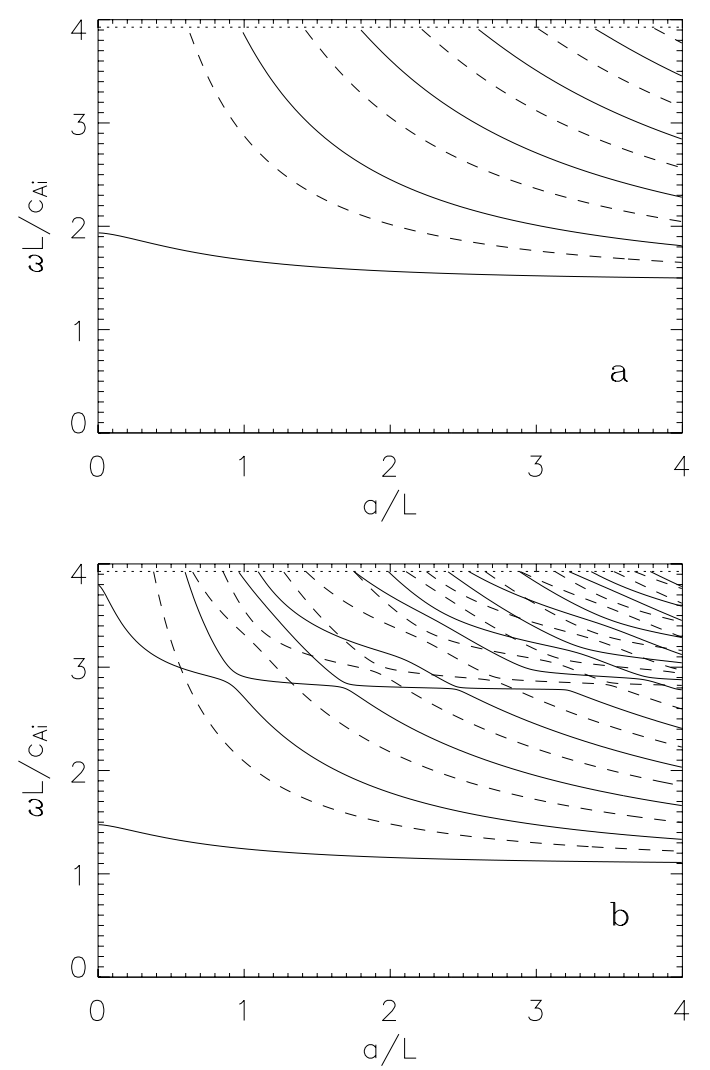

Fig. 2. Dispersion diagram plotting non-dimensional frequency $\omega L / c_{\mathrm{Ai}}$ against the non-dimensional loop radius $a / L$ for an exponentially structured loop and homogeneous environment with $c_{\mathrm{Ae}}=2.5 c_{\mathrm{Ai}}$, and a) $\rho_{\mathrm{i}}(L) / \rho_{\mathrm{i}}(0)=1.6$ or b) $\rho_{\mathrm{i}}(L) / \rho_{\mathrm{i}}(0)=8$. Sausage modes are shown as dashed curves and kink modes as solid curves; cutoffs are shown as dotted horizontal lines.

environment with $c_{\mathrm{Ae}}=2.5 c_{\mathrm{Ai}}$ and the dispersion curves of a structured loop with $\rho_{\mathrm{i}}(L) / \rho_{\mathrm{i}}(0)=8$ embedded in a uniform environment with $c_{\mathrm{Ae}}=2.5 c_{\mathrm{Ai}}$ have been overlaid. This scaling is related to the suggestion that the effect of the structure can be approximated by an unstructured loop with the same weighted mean density, weighted with the square of the fundamental eigenfunction (Andries et al. 2005b). We can perform this calculation: in the exponentially structured model (Eq. (25)), the internal weighted density $\bar{\rho}_{\mathrm{i}}$ is given by

$$
\begin{aligned}
\bar{\rho}_{\mathrm{i}} \int_{0}^{L} \cos ^{2}\left(M_{1} z\right) \mathrm{d} z & =\int_{0}^{L} \rho_{\mathrm{i}}(z) \cos ^{2}\left(M_{1} z\right) \mathrm{d} z \\
& =\rho_{\mathrm{i}}^{2}(0) \int_{0}^{L} \mathrm{e}^{2 \alpha_{\mathrm{i}} z / L} \cos ^{2}(\pi z / 2 L) \mathrm{d} z
\end{aligned}
$$

yielding

$\bar{\rho}_{\mathrm{i}}=\rho_{\mathrm{i}}(0) \frac{\pi^{2} \mathrm{e}^{2 \alpha_{\mathrm{i}}}-8 \alpha_{\mathrm{i}}^{2}-\pi^{2}}{2 \alpha_{\mathrm{i}}\left(4 \alpha_{\mathrm{i}}^{2}+\pi^{2}\right)}$

for $\rho_{\mathrm{i}}(L) / \rho_{\mathrm{i}}(0)=8$ this gives $\alpha_{\mathrm{i}}=1.04$ and $\bar{\rho}_{\mathrm{i}} / \rho_{\mathrm{i}}(0)=2.05$ and a rescaling in Fig. 3 of 1.43, which turns out to be a good approximation. However, it must be emphasised that other features, such as the second trapped mode in the thin tube limit or the avoided crossings present in Fig. 2b do not appear in this rescaling, and that the numerical agreement is not perfect, becoming worse as higher harmonics are considered.

Another interesting point is how the actual crossings of the dispersion curves of the uniform loop correspond to the avoided

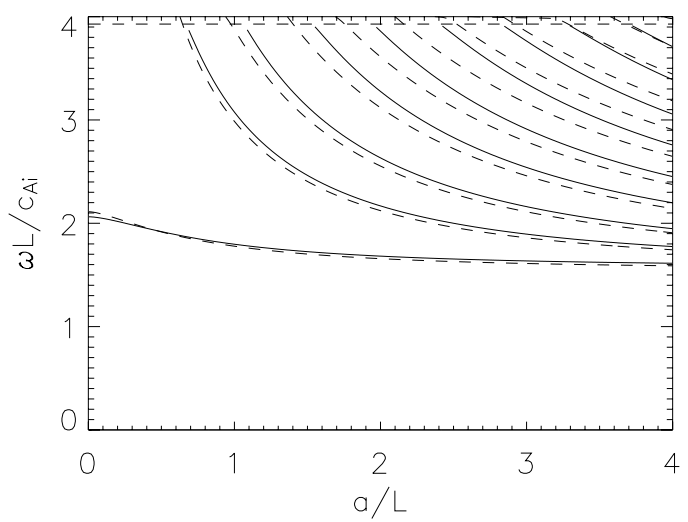

Fig. 3. Dispersion diagram plotting $\omega L / c_{\mathrm{Ai}}$ against $a / L$ for models having uniform environment with $c_{\mathrm{Ae}}=2.5 c_{\mathrm{Ai}}$. Solid lines correspond to the uniform loop of Fig. 1a (kink and sausage modes), while dashed lines correspond to the exponentially structured loop with $\rho_{\mathrm{i}}(L) / \rho_{\mathrm{i}}(0)=$ 8 , which has been rescaled by a factor 1.43 for comparison purposes.

crossings in the structured case. This suggests that for avoided crossings to occur in the structured loop the loop must have a large enough density enhancement so that a uniform loop of equivalent mass supports more than just the first family of modes below the cutoff frequency of the first family. As the oscillatory modes of a structured loop are a superposition of all families of the uniform loop, one family will generally be dominant; but at an avoided crossing the dominant family is changed, resulting in a change in spatial structure of the mode.

In conclusion, the uniform loop is a reasonable approximation to the structured loop provided an appropriate density is used. However, if loop parameters are determined from one point on the loop, especially an extreme point such as the apex or the footpoint, and the rest of the loop is assumed to be at the same value, this will give a poor representation, since some sort of averaging is necessary. If a higher accuracy is required, then the structured model should be employed rather than the uniform model, if the model is to capture all features such as modified frequencies and spatial structure of the eigenfunctions and interactions between modes at avoided crossings.

\subsubsection{Dependence on the footpoint density}

We next investigate the dependence of the oscillation frequency on the strength of the structure. This is displayed in Fig. 4, showing dispersion diagrams of the non-dimensional frequency as a function of the longitudinal density contrast $\rho_{\mathrm{i}}(L) / \rho_{\mathrm{i}}(0)$ between the loop base and the loop apex. We consider a thin tube, $a / L=0.01$, which is in line with the value used by Nakariakov et al. (1999). In the uniform loop case $\left(\rho_{\mathrm{i}}(L) / \rho_{\mathrm{i}}(0)=1\right)$ and in the thin tube limit, only the fundamental kink mode propagates as a trapped mode. For moderate levels of structuring $\left(\rho_{\mathrm{i}}(L) / \rho_{\mathrm{i}}(0)=1-20\right)$ it is seen that the frequency of the fundamental kink mode is strongly dependent on the density structure, causing the frequency to decrease rapidly, this decrease becoming slower as $\rho_{\mathrm{i}}(L) / \rho_{\mathrm{i}}(0)$ becomes large.

Since the addition of density to the loop results in a reduction in oscillation frequency, eventually many of the kink harmonics (as well as the fundamental) will propagate as trapped modes in the thin tube limit. However, this is not the case for the sausage mode which never propagates as a trapped mode in the thin tube limit. 


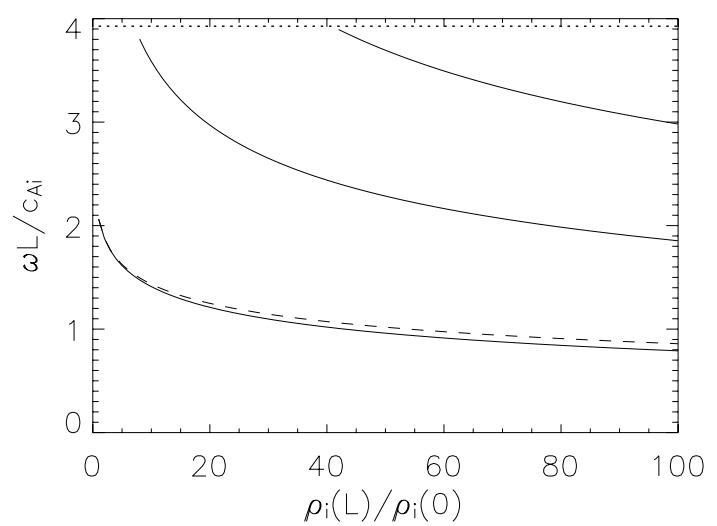

Fig. 4. Dispersion diagram plotting as solid lines the non-dimensional frequency $\omega L / c_{\mathrm{Ai}}(0)$ against the longitudinal density ratio $\rho_{\mathrm{i}}(L) / \rho_{\mathrm{i}}(0)$ for an internal density with $c_{\mathrm{Ae}}(z=0)=2.5 c_{\mathrm{Ai}}(z=0)$ and $a / L=0.01$. Overplotted in a dashed line is the result of a uniform loop (Eq. (24)) with the averaged density given in Eq. (33).

In Fig. 4, the results for the fundamental mode of a uniform loop - as determined by Eq. (24) with weighted averaged density given by Eq. (33) - have been overplotted to highlight that the average profile produces reasonably accurate results for the fundamental mode.

\subsection{Structured environment}

In order to isolate the effect of structure in the environment of a coronal loop we consider an exponentially structured environment surrounding a homogeneous loop, providing an extension of the study carried out in Donnelly et al. (2006). In this case the density of the environment increases towards the chromospheric-photospheric base and so can become denser than the loop. As the overall density of the environment has increased relative to the loop, the loop becomes a less efficient waveguide supporting fewer trapped modes. As noted in Donnelly et al. (2006), environmental structure reduces the cutoff frequency of the trapped modes, enhancing the leakage.

Figure 5 shows the dispersion diagram for a homogeneous loop in a structured environment with $\rho_{\mathrm{e}}(L) / \rho_{\mathrm{e}}(0)=10$ or $\rho_{\mathrm{e}}(L) / \rho_{\mathrm{e}}(0)=50$, with Fig. 1a overplotted for comparison purposes. The diagram shows similarities with that of the uniform loop in a uniform environment, with the fundamental kink mode being the only mode to propagate for all $a / L$; all other modes reach a uniform cutoff frequency beyond which the modes become leaky. All modes have a dispersive nature so their frequency is strongly dependent on the loop radius or length. However, in the realistic thin tube region $\left(a / L=10^{-3}-10^{-1}\right)$, only the fundamental kink mode propagates and then $\omega L / c_{\mathrm{Ai}}$ is insensitive to the loop radius. Comparing the frequencies for structured environment with those of the unstructured environment, we note that there is a significant reduction in the cutoff frequency from $\omega_{\text {cut }} L / c_{\mathrm{Ai}} \approx 3.9$ for a uniform environment to $\omega_{\text {cut }} L / c_{\mathrm{Ai}} \approx 2.6$ and $\omega_{\text {cut }} L / c_{\mathrm{Ai}}=1.7$ for the exponential environments with $\rho_{\mathrm{e}}(L) / \rho_{\mathrm{e}}(0)=10$ and $\rho_{\mathrm{e}}(L) / \rho_{\mathrm{e}}(0)=50$, respectively. As a result of the reduced cutoff frequency, the value of $a / L$ for which modes become leaky is increased. Another interesting point is that the modification to the frequency in the range where both sets of modes are trapped is relatively small, especially for larger values of $a / L$. Notice also that the structure has resulted in a reduction in the dispersive effect of $a / L$ when compared with the uniform environment.

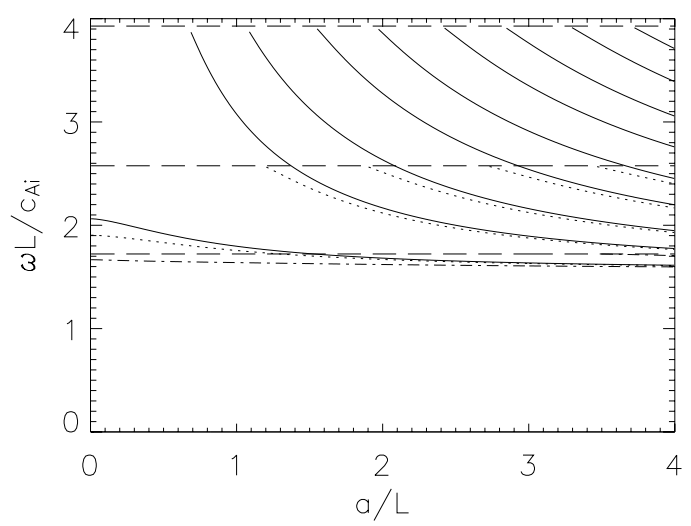

Fig. 5. Dispersion diagram plotting $\omega L / c_{\mathrm{Ai}}$ against $a / L$ for homogeneous loops in a structured environment, with $c_{\mathrm{Ae}}(z=0)=2.5 c_{\mathrm{Ai}}(z=0)$. Solid lines are the frequencies for a homogeneous environment $\left(\rho_{\mathrm{e}}(L) / \rho_{\mathrm{e}}(0)=1\right)$, dotted lines for an exponentially structured environment with $\rho_{\mathrm{e}}(L) / \rho_{\mathrm{e}}(0)=10$, and dot-dashed lines for an exponentially structured environment with $\rho_{\mathrm{e}}(L) / \rho_{\mathrm{e}}(0)=50$. The cutoff for each model has been overplotted as a horizontal dashed line.

We conclude that environmental structure results in a small modification to the oscillation frequency and a reduction in the dispersive nature of the modes. The most significant alteration to the modes due to environmental structure occurs in the cutoff frequency: adding density results in a reduction in the cutoff frequency and so leakage is enhanced. It should be noted that density structuring with $\rho_{\mathrm{e}}(L) / \rho_{\mathrm{e}}(0)=10$ or 50 are modest compared with a more realistic value of $\rho_{\mathrm{e}}(L) / \rho_{\mathrm{e}}(0)=100$, but trapped modes no longer propagate for such high values.

We next consider the influence of varying the level of structuring in the environment to examine the effect on the oscillation frequency and the cutoff frequency. We can see these effects in Fig. 6. As previously noted, the external structure has little influence on the oscillation frequency in the thin tube case, but its main impact is on the cutoff frequency, in that the addition of density to the environment in comparison with the loop results in a reduction in the cutoff frequency as the loop becomes a less efficient wave guide. In Fig. 6 we can see a significant reduction in cutoff frequency, from a value of $\omega_{\text {cut }} L / c_{\mathrm{Ai}}=3.9$ in the uniform case to a value of $\omega_{\text {cut }} L / c_{\mathrm{Ai}}=1.4$ for a structuring of $\rho_{\mathrm{e}}(L) / \rho_{\mathrm{e}}(0)=100$. Notice the most rapid reduction in cutoff frequency occurs as low levels of structuring are introduced, $\rho_{\mathrm{e}}(L) / \rho_{\mathrm{e}}(0)=1-10$, and the gradient becomes shallower as $\rho_{\mathrm{e}}(L) / \rho_{\mathrm{e}}(0)$ becomes larger. Another feature of the cutoff frequency is that it is independent of the loop radius. As a result of the reduction in the cutoff frequency, modes that have previously been considered to propagate as a trapped mode, such as the fundamental kink mode in the thin tube limit, are now seen to reach the cutoff frequency and become leaky. Also in thicker loops, where many sausage and kink modes are expected to propagate as trapped modes, for large enough environmental structuring (say $\rho_{\mathrm{e}}(L) / \rho_{\mathrm{e}}(0) \geq 70$ ) all modes become leaky.

\subsection{Structure in both loop and environment}

Having seen separately the effects of structure in the loop or in the environment, we now combine the effects of longitudinal structure both inside and outside the loop, thus producing a more realistic model for a coronal loop. We have seen that the main influence of the internal structure is to modify the oscillation frequency and the main influence of the external structure 


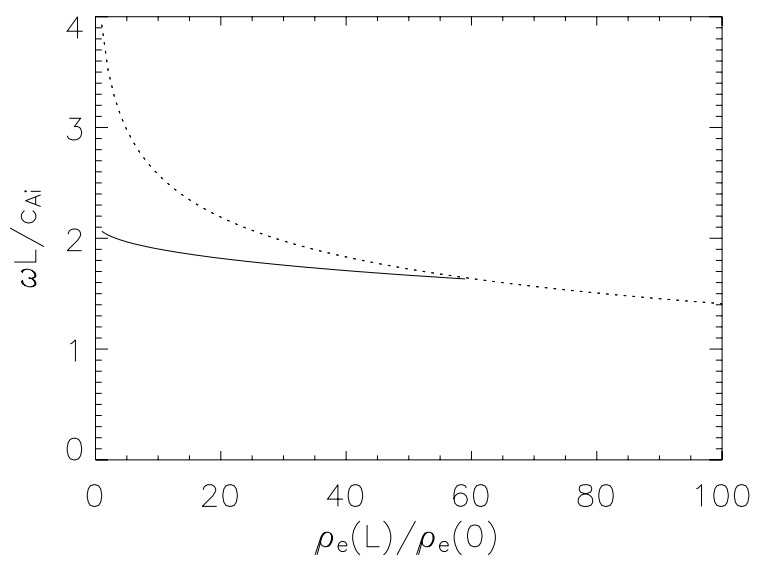

Fig. 6. Dispersion diagram for a uniform loop in a exponentially structured environment, plotting $\omega L / c_{\mathrm{Ai}}(0)$ against the external density structuring $\rho_{\mathrm{e}}(L) / \rho_{\mathrm{e}}(0)$, for $c_{\mathrm{Ae}}(0)=2.5 c_{\mathrm{Ai}}(0)$ and a loop radius of $a / L=0.01$.

is in a modification in the cutoff frequency. We consider a realistic density ratio in the environment of $\rho_{\mathrm{e}}(L) / \rho_{\mathrm{e}}(0)=100$ between the coronal and chromospheric-photospheric levels, and assume the photosphere to be uniform so that $\rho_{\mathrm{e}}(L)=\rho_{\mathrm{i}}(L)$. Although this may not always be accurate, we do not expect significant changes due to variations in density in the lower atmosphere, especially since the perturbations fall to zero at the footpoints to satisfy the line tying condition. Hence, choosing again $c_{\mathrm{Ae}}(0)=2.5 c_{\mathrm{Ai}}(0)$ results in $\rho_{\mathrm{i}}(L) / \rho_{\mathrm{i}}(0)=16$.

The dispersion diagram for this case is shown in Fig. 7 and is very similar in structure and appearance to the uniform loop and uniform environment: the fundamental kink mode propagating for all $a / L$ and other modes reach the cutoff frequency. Also kink and sausage modes are again distributed alternately and there are no avoided crossings present. The most interesting aspects are seen most clearly when we make direct comparison between this case and the homogeneous loop and environment, where we have simply matched the densities at the loop apex. We see immediately that the fast mode bands for these two different coronal loop structures do not overlap. The structuring of the corona has reduced the dimensionless cutoff frequency to 1.4 (also see Fig. 6), which is below the lower limit of the frequency for the uniform loop and environment. Also the value of $a / L$ for which modes reach the cutoff frequency has increased as a result of the reduction in the cutoff frequency. Previously, when we implemented such a large environmental structuring around a uniform loop, we found that the loop did not support any trapped modes. However, as noted earlier, the introduction of internal structure results in a modification to the oscillation frequency and in this case the loop again supports many trapped modes.

Therefore, we conclude exponential structuring of the form in Eq. (25) is an important factor for the cutoff frequency but more importantly for the oscillation frequency and period of the modes. If a uniform interior and exterior are used to approximate a structured loop, it is important that the parameters chosen for the uniform case reflect the overall structure along the loop rather than simply matching the densities at a single point (such as the apex or the footpoint). In fact the apex or the footpoints are inappropriate points to choose for a comparison, since they are most extreme in the model. A more effective method of representing the structured case by a uniform case is to match the total integrated densities in each of the appropriate regions.

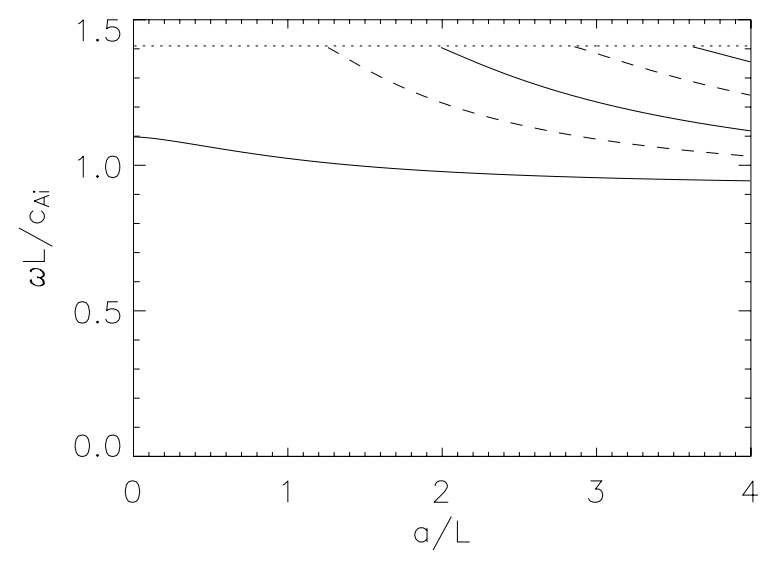

Fig. 7. Dispersion diagram plotting $\omega L / c_{\mathrm{Ai}}$ against the $a / L$ for a) an exponentially structured loop and environment with $c_{\mathrm{Ae}}(z=0)=$ $2.5 c_{\mathrm{Ai}}(z=0), c_{\mathrm{Ae}}(z=L)=c_{\mathrm{Ai}}(z=L)$ and $\rho_{\mathrm{e}}(L) / \rho_{\mathrm{e}}(0)=100$.

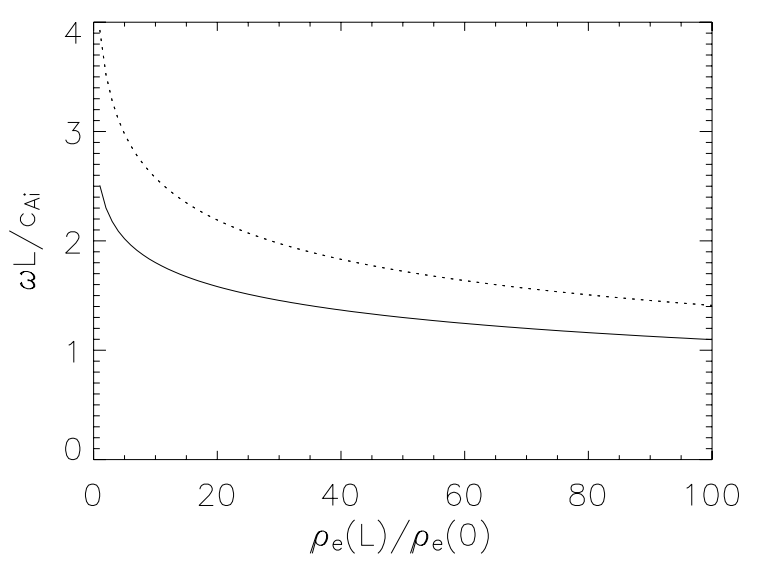

Fig. 8. Dispersion diagram plotting $\omega L / c_{\mathrm{Ai}}$ against density structuring $\rho_{\mathrm{e}}(L) / \rho_{\mathrm{e}}(0)$ for $a / L=0.01$ and $c_{\mathrm{Ae}}(z=0)=2.5 c_{\mathrm{Ai}}(z=0)$, with an uniform base $\left(\rho_{\mathrm{e}}(L)=\rho_{\mathrm{i}}(L)\right)$.

Next we allow the external density structuring to vary, but retain the assumption that the loop footpoint $(z= \pm L)$ is uniform (so $\rho_{\mathrm{e}}(L)=\rho_{\mathrm{i}}(L)$ ). The internal structure is modified appropriately. For this case the loop always has a density enhancement over its environment. Figure 8 shows the dispersion diagrams plotting non-dimensional frequency against $\rho_{\mathrm{e}}(L) / \rho_{\mathrm{e}}(0)$. The cutoff curve is identical to that of Fig. 6 , since it is independent of the internal structure and loop radius. We can see that in both cases modes remain trapped for a larger range of density structuring compared with the case displayed in Fig. 6. This is due to the modification of the oscillation frequency as a result of the internal loop structure, where we can see the fundamental kink mode remains trapped for all values of structuring shown in Fig. 8 for the thin tube limit. Therefore, in this case the effect of internal structuring in modifying the oscillatory frequency is greater than the effect of the external structuring in reducing the cutoff frequency.

\subsection{Exponentially structured chromosphere}

In the previous section we took a step forward in that the density structuring of the loop is continuous, whereas in Díaz et al. (2004) and Donnelly et al. (2006) step functions were used to represent the longitudinal density profile changing from the coronal to photospheric values. We consider a uniform coronal region but allow an exponential density profile in the 


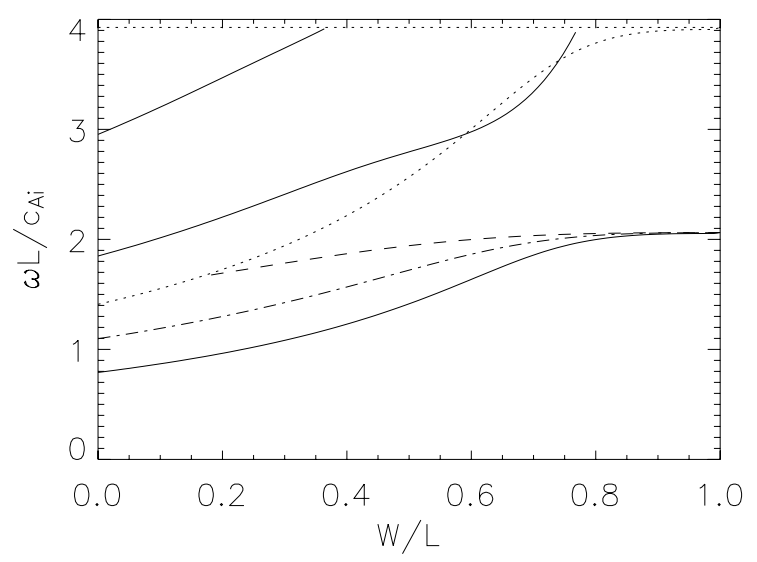

Fig. 9. Dispersion diagram plotting $\omega L / c_{\mathrm{Ai}}$ against the fractional coronal depth $W / L$ for $a / L=0.01, \rho_{\mathrm{i}}(L) / \rho_{\mathrm{i}}(0)=16$ and $c_{\mathrm{Ae}}(0)=2.5 c_{\mathrm{Ai}}(0)$. The solid lines are the modes of a loop with chromospheric layers embedded in a uniform environment (whose cutoff is the horizontal straight line); the dashed line is the only propagating mode of a uniform loop embedded in an environment with chromospheric layers, while the dotdashed line corresponds to a loop with chromospheric layers both inside and outside. The cutoff of these last two models has been overplotted as a dotted line.

chromospheric layers of thickness $L-W$ near the footpoints. Thus, the profile of the Alfvén speed is

$c_{\mathrm{A}}(z)=\left\{\begin{array}{lc}c_{\mathrm{Aj}} \exp \left(\alpha_{\mathrm{j}}(z+W) / L\right), & -L<z<-W, \\ c_{\mathrm{Aj}}, & -W<z<W, \\ c_{\mathrm{Aj}} \exp \left(-\alpha_{\mathrm{j}}(z-W) / L\right), & W<z<L .\end{array}\right.$

The corresponding $h(z)$ functions from Eq. (9) are

$h(z)=\left\{\begin{array}{l}C_{1} F_{i v}(x)+D_{1} G_{i v}(x), \quad-L<z<-W, \\ C_{2} \sin k z+D_{2} \cos k z, \quad-W<z<W, \\ C_{3} F_{i v}(x)+D_{3} G_{i v}(x), \quad W<z<L,\end{array}\right.$

with $C_{1}, C_{2}, C_{3}, D_{1}, D_{2}$ and $D_{3}$ being arbitrary constants.

If we select the density profile inside the loop consistent with Eq. (34) and assume a uniform environment there is a modification in the oscillation frequency but not the cutoff frequency. However, the introduction of structuring across the chromospheric region results in a slight correction to the frequency of the uniform loop. We consider the impact of varying the chromospheric layer thickness $(L-W)$ while holding the internal density ratio fixed at $\rho_{\mathrm{i}}(L) / \rho_{\mathrm{i}}(0)=16$. Figure 9 show dispersion diagrams for this model. The case $W / L=0$ corresponds to the exponential loop profile (Eq. (25)) considered earlier and $W / L=1$ gives the uniform loop, so we will be able to observe the evolution from the structured case to the uniform case. Figure 9 shows that only the fundamental kink mode is able to propagate as a trapped mode for all values of $W / L$, and its frequency increases as we evolve from the exponential structure to the uniform loop. Also, for smaller values of $W / L$ we find the first and second kink harmonics are trapped in the thin tube limit but are seen to reach the cutoff frequency before the realistic range of values of $W / L \approx 0.8-1.0$. Importantly, for a realistic range of chromospheric depth, the frequency of the fundamental mode is insensitive to the chromospheric depth. We conclude that loop structuring of the form (34) does not cause significant changes to the frequency for realistic parameters $(W / L=0.8-1.0)$. In fact, it is reasonable to assume a uniform loop provided it is chosen appropriately to match the structured loop at the apex.

On the other hand, if we isolate the effect of structure of the form of Eq. (34) in the environment by considering a uniform loop we obtain a modification to the cutoff frequency in this case, but only a small correction to the oscillatory frequency. Again we investigate the influence of the parameter $W / L$ on the oscillation and cutoff frequencies. This is overplotted in Fig. 9 as a dotted line. Only the fundamental kink mode propagates as a trapped mode, and even this mode has a cutoff for $W / L \geq 0.2$ (similar to Fig. 6). The oscillation frequency of the fundamental kink mode shows only a slight dependence on $W / L$, especially in the realistic range of $W / L=0.8-1.0$.

So far we have explored the effects of structuring in density by looking separately at its role in the loop interior or in the loop environment. In reality, of course, the effects will arise in a combined way. Structure is again taken to be of the form of Eq. (34), now applied in both regions. In the environment, we choose $\rho_{\mathrm{e}}(L) / \rho_{\mathrm{e}}(0)=100$; however, we assume a uniform photospheric base, so $\rho_{\mathrm{e}}(L)=\rho_{\mathrm{i}}(L)$. The implication of this is that the density structure of the loop across the chromosphere depth is less than that of the environment. The structuring results in a small correction to the oscillation frequency, which is mainly due to the internal structure. However, the reduction in the cutoff frequency is entirely a result of the external structuring. Again, the uniform case provides an adequate representation of this loop structure and would thus serve adequately for the purpose of coronal seismology, but care should be taken in using modes close to the cutoff. However, in this case the fundamental kink mode does not reach the cutoff frequency in the thin tube case (Fig. 9, dot-dashed line). This is due to the reduction in its frequency as a result of the internal structuring as $W / L \rightarrow 0$ (such as in Fig. 8). The frequency of all trapped modes in the realistic range where $W / L=0.8-1.0$ is found to be independent of $W / L$. This again points to the fact that the uniform loop and uniform environment provides a robust model.

We can also explore the case in which the footpoint density $\rho_{\mathrm{i}}(L)$ becomes relatively large. In such a limit, $\alpha_{\mathrm{i}}$ becomes large, and the coefficients of the Bessel functions of imaginary order in Eq. (35) become small. These functions have the property of having an infinite number of oscillations as $x \rightarrow 0$ (Dunster 1990), so other modes would be able to propagate under that limit: those which a number of small wiggles in the chromospheric regions $(W<|z|<L)$. This type of solutions have been described for the slow mode in Dymova \& Ruderman (2006) and McEwan et al. (2006). However, these solutions appear under the cutoff for extremely large values of $\rho_{\mathrm{i}}(L) / \rho_{\mathrm{i}}(0)$.

\section{Period ratios}

There is some observational evidence (Verwichte et al. 2004; Van Doorsselaere et al. 2007) that the period ratio $P_{1} / n P_{n}$ between the period $P_{1}$ of the fundamental kink mode and its harmonics with period $P_{n}, n \geq 2$ deviates from unity in solar arcades. Although there are only a few detections of harmonics in coronal loops, the potential of this ratio for coronal seismology has been studied theoretically (Andries et al. 2005a; McEwan et al. 2006, 2007).

In McEwan et al. (2006) various factors that cause the period ratio to deviate from unity were discussed, and it was found that the most important one was the effect of longitudinal structure. Here we calculate the ratio $P_{1} / 2 P_{2}$ for the continuous exponential density profile of Sect. 4.1. The results are shown in Fig. 10 for two values of $\rho_{\mathrm{i}}(L) / \rho_{\mathrm{i}}(0)$, pointing out that for values of $a / L<10^{-1}$, the longitudinal structuring is mainly responsible of the shift.

Therefore, it is interesting to take a fixed value of $a / L$ and study the dependence of the ratio $P_{1} / 2 P_{2}$ as a function of the 


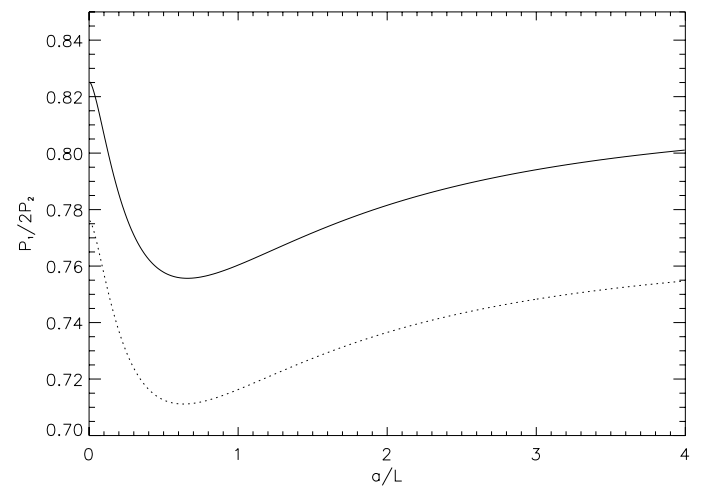

Fig. 10. $P_{1} / 2 P_{2}$ as a consequence of longitudinal structuring. The density is exponentially stratified along the loop, the solid line has a base density that is 8 times the density at the apex, $\rho_{\mathrm{i}}(L) / \rho_{\mathrm{i}}(0)=8$, and the dotted line has a base density that is 16 times the density at the apex, $\rho_{\mathrm{i}}(L) / \rho_{\mathrm{i}}(0)=16$. The tube is structured radially so that $c_{\mathrm{ae}}(0)=$ $2.5 c_{\mathrm{Ai}}(0)$, corresponding to a tube density enhancement at the apex of $25 / 4$ times the environment density at the apex.

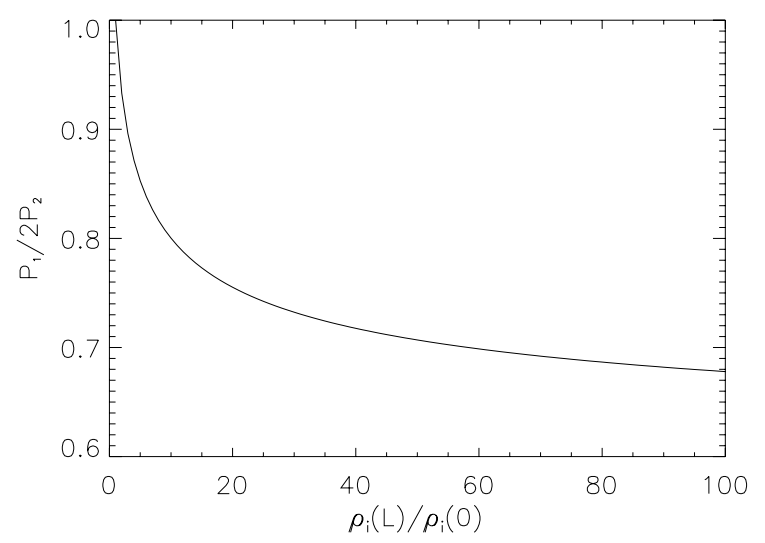

Fig. 11. $P_{1} /\left(2 P_{2}\right)$ as a function of the density contrast $\rho_{\mathrm{i}}(L) / \rho_{\mathrm{i}}(0)$ for a coronal loop structured exponentially in density. Here we have taken $a / L=10^{-3}$ and $c_{\mathrm{Ae}}(0)=2.5 c_{\mathrm{Ai}}(0)$.

density contrast (Fig. 11). The result is a monotonically decreasing function. These results can be used in coronal seismology to estimate the scale height of the model (see McEwan et al. 2006).

Using the results of Sect. 4.3, we could find a similar plot if an exponential density profile is considered in the embedding corona. We checked that the main effect is to modify the cutoff frequency, but for typical parameters the fundamental mode and its first harmonic do not reach it, and the frequency shift is mainly because of the internal structure. A plot very similar to Fig. 11 results.

On the other hand, the ratio $P_{1} / 3 P_{3}$ between the fundamental mode and its first even harmonic might also be of interest. In a homogeneous medium this ratio is unity, while in a coronal arcade it is no longer monotonically decreasing because of the appearance of avoided crossings between modes with the same symmetry (Donnelly et al. 2007). Avoided crossings are also present in the loop modes (Fig. 2b), so the $P_{1} / 3 P_{3}$ has extrema, shown in Fig. 12 for a coronal loop in a homogeneous corona (dashed line). However, in a coronal loop the first even harmonic becomes leaky for small values of $a / L$ (Figs. 2a and 7), so it is less useful as a seismological tool, since the leaky modes are much harder to detect. We can see it in Fig. 12 (solid line), which shows that the mode becomes leaky further from the

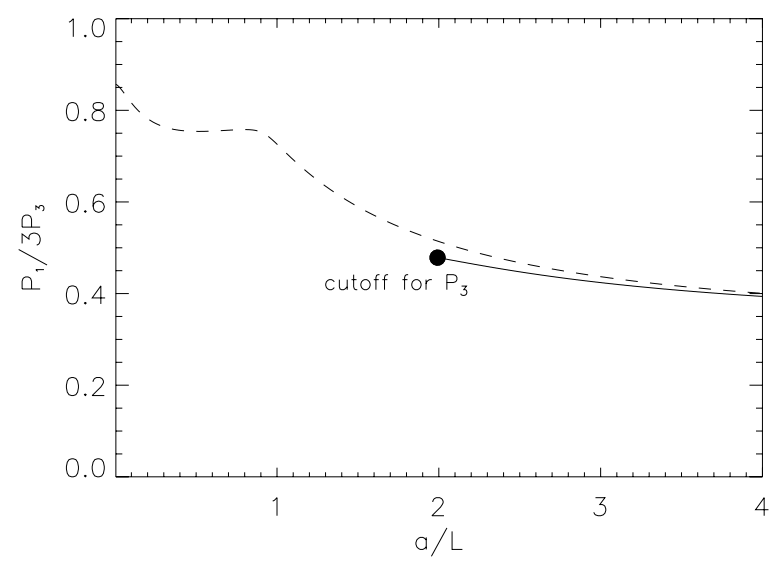

Fig. 12. $P_{1} /\left(3 P_{3}\right)$ as a function of $a / L$ for a structured loop with $\rho_{\mathrm{i}}(L) / \rho_{\mathrm{i}}(0)=8$ for two different surrounding media: a homogeneous corona with $c_{\mathrm{Ae}}(0)=2.5 c_{\mathrm{Ai}}(0)$ (dashed line) and a structured corona with $c_{\mathrm{Ae}}(0)=2.5 c_{\mathrm{Ai}}(0)$ and $\rho_{\mathrm{e}}(L) / \rho_{\mathrm{e}}(0)=8$ (solid line). The point where the second kink harmonic has a cutoff has been labelled in the plot.

typical values of $a / L$ for coronal loops. It is important to remark that the coronal structuring plays a relevant role in this ratio, since we can see in Fig. $2 b$ that if the coronal structuring is not considered, then these harmonics may become trapped so $P_{1} / 3 P_{3}$ can be obtained for all the values of $a / L$, but this is a mathematical artifact due to that assumption of a homogeneous corona.

\section{Conclusions}

We have studied the effect of longitudinally structured density profiles in either the loop interior, its exterior, or both. This extends the results in Donnelly et al. (2006), where uniform layers were used. The first of these profiles was was a purely exponential one and so the transition between the maximum density at the loop footpoint and minimum at the apex is gradual, and therefore does not reflect the more realistic rapid change from the photosphere to the corona over the height of the chromosphere. For this profile the frequency of the oscillation is strongly dependent on $\rho_{\mathrm{i}}(L) / \rho_{\mathrm{i}}(0)$, the ratio of the loop apex density to density at the footpoints. This strong dependence reflects the large addition of mass in the loop associated with the exponential profile. Comparisons with a uniform loop show poor agreement in frequency when the uniform loop parameters are chosen to match the apex of the structured loop. However, a much better agreement is observed when a weighted average for the density is used, as suggested by Andries et al. (2005b). When the structure of the environment is included a modification in the cutoff frequency arises; comparatively small changes in the oscillation frequency also occur, when compared with the effect of internal structure. Drawing comparison with the uniform loop in the case of the internal structure, good agreement is found when the profiles of the structured and uniform environments are in mass balance.

The second density profile we considered was chosen to represent more accurately the rapid transition between the photosphere and the corona, extending the results for piecewise functions in Díaz et al. (2004) and Donnelly et al. (2006). In this profile a uniform coronal region of extent $2 W$ is assumed to be grounded by an exponentially structured chromosphere. For this case there is excellent agreement between the uniform loop and structured loop which are matched at the apex. Other effects 
that appeared in previous piecewise works are also present here, namely, the avoided crossings between modes and the apparition of small extrema in the dense regions near the footpoints of the loop. Regarding the frequency of the fundamental modes of each family, these results demonstrate the robust nature of the Edwin \& Roberts (1983) model and gives confidence to the results of coronal seismology (see, for example, Nakariakov \& Ofman 2001) based on this model. Nonetheless, if observational data of sufficient accuracy were available, it should prove possible to probe structure effects through the use of more complex models such as that investigated here.

The ratio between different harmonics has also proven to contain valuable information about the structure, and it is ready to be used in coronal seismology. We have calculated the deviation from unity in $P_{1} / 2 P_{2}$, and these results were used in McEwan et al. (2006, 2007) to estimate the loop scale height. Although choosing different equilibrium density profiles leads to some differences in this ratio (Dymova \& Ruderman 2006), Fig. 10 gives a good diagnostic tool for a generic loop. On the other hand, the information of these ratios for higher harmonics might prove less useful, since these modes are leaky for typical values of $a / L$, and therefore harder to detect observationally.

In conclusion, the extension of this work for continuous density profiles confirms that internal longitudinal structure shifts the phase speed of the fundamental kink mode from $c_{\mathrm{k}}$ in a way that can be explained by considering a uniform loop with weighted averaged density, while external structure shifts the cutoff frequency. For typical coronal parameters, the fundamental mode is still able to propagate with its frequency shifted.

Acknowledgements. The authors acknowledge financial support from PPARC. AJD also acknowledges financial support from the Spanish government Grant No. AYA2006-07637.

\section{References}

Abramowitz, M., \& Stegun, I. A. 1964, Handbook of Mathematical Functions (National Bureau of Standards)

Andries, J., Arregui, I., \& Goossens, M. 2005a, ApJ, 624, L57

Andries, J., Goossens, M., Hollweg, J. V., Arregui, I., \& Van Doorsselaere, T. 2005b, A\&A, 430, 1109

De Moortel, I., Hood, A. W., \& Ireland, J. 2002, A\&A, 381, 311

Díaz, A. J. 2004, Ph.D. Thesis, Departament de Física, Universitat de les Illes Balears, Palma Mallorca

Díaz, A. J., Oliver, R., Erdélyi, R., \& Ballester, J. L. 2001, A\&A, 379, 1083

Díaz, A. J., Oliver, R., \& Ballester, J. L. 2002, ApJ, 580, 550

Díaz, A. J., Oliver, R., Ballester, J. L., \& Roberts, B. 2004, A\&A, 424, 1055

Díaz, A. J., Oliver, R., \& Ballester, J. L. 2006, ApJ, 645, 766

Donnelly, G. R., Díaz, A. J., \& Roberts, B. 2006, A\&A, 457, 707

Donnelly, G. R., Díaz, A. J., \& Roberts, B. 2007, A\&A, 471, 999

Dunster, T. M. 1990, Siam. J. Math. Anal., 21, 995

Dymova, M. V., \& Ruderman, M. S. 2006, A\&A, 457, 1059

Edwin, P. M., \& Roberts, B. 1983, Sol. Phys., 88, 179

Erdélyi, R., \& Verth, G. 2007, A\&A, 462, 743

Goedbloed, J. P. 1983, Lecture Notes on Ideal Magnetohydrodynamics (Rijnhuizen Report), 76

Hood, A. W. 1986, Sol. Phys., 105, 307

James, L. 2003, Master's thesis, School of Mathematics \& Statistics, University of St Andrews

McEwan, M. P., Donnelly, G. R., Díaz, A. J., \& Roberts, B. 2006, A\&A, 460, 893

McEwan, G. R., Díaz, A. J., \& Roberts, B. 2007, A\&A, in press

Mendoza-Briceño, C. A., Erdélyi, R., \& Sigalotti, L. D. G. 2004, ApJ, 605, 493

Nakariakov, V. M., \& Ofman, L. 2001, A\&A, 372, L53

Nakariakov, V. M., Ofman, L., Deluca, E. E., Roberts, B., \& Davila, J. M. 1999, Science, 285, 862

Nakariakov, V. M., Verwichte, E., Berghmans, D., \& Robbrecht, E. 2000, A\&A, 362,1151

Roberts, B. 1986, in Proc. NASA. Goddard Space Flight Center Coronal and Prominence Plasmas, 325

Roberts, B., Edwin, P. M., \& Benz, A. O. 1984, ApJ, 279, 857

Van Doorsselaere, T., Nakariakov, V. M., \& Verwichte, E. 2007, A\&A, 473, 959

Verwichte, E., Nakariakov, V. M., Ofman, L., \& Deluca, E. E. 2004, Sol. Phys., 223,77 